Results 3 polymorphisms (A46G, C79G and A523C) and a mutation (C491T) of ADRB2 were found. Genotype distributions of A523C in control and hypertriglyceridemia, control and hypo-HDL-c individuals were different $(p<0.05)$. Logistic regression analysis showed that the OR for hypertension was 2.894 in C523C group against the $A 523 A+A 523 C$ group $(p=0.009)$; compared the A523A+A523C group, the OR for hypertriglyceridemia in C523C group were 2.666 $(p=0.031)$. The frequency of C491T was $1.2 \%(n=5)$ and no TT genotype was found. That C491T was found only in hypertensive or dyslipidemia group. All five subjects were diagnosis with hypertension and dyslipidemia. Four out of five individuals with C491T had Hyper-LDL-C. The mean blood pressure and serum LDL-C of the five subjects were much higher than normal value.

Conclusions Variation of ADRB2 may play a causal role in the pathogenesis of the hypertension and dyslipidemia in Xinjiang Kazakans.

\section{e0167 THE RELATIONSHIP STUDY BETWEEN T663A POLYMORPHISM OF QENAC GENE WITH ESSENTIAL HYPERTENSION AND SERUM ELECTROLYTES IN XINJIANG KAZAKHS}

doi:10.1136/hrt.2010.208967.167

Li Nanfang, Xu Hong, Yao Xiaoguang, Yan Zhitao. Hypertension Unit of The People's Hospital of Xinjiang Uygur Autonomous Region, The Institute of Hypertension of Xinjiang, The Center of Hypertension of The People's Hospital of Xinjiang Uygur Autonomous Region, Urumqi, China

Introduction To study the relationship between the T663A polymorphism in $\alpha \mathrm{ENaC}$ gene with essential hypertension $(\mathrm{EH})$ and serum electrolytes of Xinjiang Kazakhs.

Methods 516 Xinjiang Kazakhs aged elder than 30 years were recruited in this population based on case-control study from the patural area of Xinjiang, and were divided into hypertensives $(\mathrm{EH}$ group) and normotensives (NT group). The gene polymorphism of T663A by PCR-RFLP and the serum electrolytes were examined.

Results The frequencies of AA, AG, GG genotypes were $15.7 \%$, $50.2 \%, 34.1 \%$ and the frequencies of alleles $\mathrm{A}, \mathrm{G}$ were $40.8 \%, 59.2 \%$, respectively. The frequencies of AA, AG, GG were $16.3 \%, 49.1 \%$, $34.6 \%$ in $\mathrm{EH}$ group and $15.1 \%, 51.4 \%, 33.5 \%$ in NT group, respectively. The distribution of genotypic and allelic frequencies were not different between $\mathrm{EH}$ group and NT group $(\mathrm{p}=0.85 ; \mathrm{p}=1.0)$. No significant difference in levels of blood pressure and $\mathrm{K}^{+}, \mathrm{Na}^{+} / \mathrm{K}^{+}$ between subjects among genotypes. The T663A polymorphism were excluded as independent variables controlling age and BMI. But the serum $\mathrm{Na}^{+}$of the subjects with AA genotype was higher than those subjects with AG and GG genotypes $(p=0.032)$.

Conclusions T663A polymorphism might not be associated with $\mathrm{EH}$ but the AA genotype of T663A polymorphisms might be associated with the higher level of the serum $\mathrm{Na}^{+}$of Xinjiang Kazakhs.

\section{e0168 THE RELATIONSHIP STUDY BETWEEN T3593C POLYMORPHISM OF $\alpha$ ENAC GENE WITH ESSENTIAL HYPERTENSION AND SERUM ELECTROLYTES IN XINJIANG KAZAKHS}

doi:10.1136/hrt.2010.208967.168

Li Nanfang, Xu Hong, Yan Zhitao, Wang Hongmei. Hypertension Unit of The People's Hospital of Xinjiang Uygur Autonomous Region, The Institute of Hypertension of Xinjiang, The Center of Hypertension of The People's Hospital of Xinjiang Uygur Autonomous Region, Urumqi, China

Introduction To study the relationship between T3593C polymorphism in the $\alpha$ subunit gene of epithelial sodium channel
$(\mathrm{ENaC})$ with essential hypertension $(\mathrm{EH})$ and serum electrolytes of Xinjiang Kazakhs.

Methods More than 500 Xinjiang Kazakhs aged more than 30 years were recruited by cluster sampling from the pasture area of Xinjiang and were divided into hypertensives (EH group) and normotensives (NT group). The genotypes of T3593C were determined by PCRRFLP method and the serum electrolytes were measured.

Results The frequencies of TT, TC, CC genotypes were $88.39 \%$, $10.63 \%, 0.98 \%$ and the alleles frequencies of $\mathrm{T}$, C were $93.7 \%, 6.3 \%$, respectively. The distributed genotypic frequencies of TT, TC, CC were $89.33 \%, 9.88 \%, 0.79 \%$ in $\mathrm{EH}$ group and $87.45 \%, 11.37 \%, 1.18 \%$ in NT group, respectively. The genotypic and allelic frequencies were not different between $\mathrm{EH}$ group and NT group ( $p=0.78 ; p=0.46)$. There was no significant difference in levels of blood pressure and serum electrolytes between subjects with the TT and TC+CC genotypes ( $p>0.05$ ). The T3593C polymorphism were excluded as independent variables which related to the blood pressure and serum electrolytes of subjects controlling FPR age and gender by multiple logistic analysis. Conclusions The T3593C polymorphism might not be associated with EH and serum electrolytes of Xinjiang Kazakhs.

\section{e0169 IN VITRO EFFECTS OF LOW MOLECULAR WEIGHT HEPARIN ON CLOT RATE}

doi:10.1136/hrt.2010.208967.169

Shi Xu-bo, Zheng Hua, Biao Hong, Li Chao, Yang Yi. Department of Cardiology, Beijing Tong Ren Hospital, Beijing, China

Objective This study was designed to determine the in vitro effects of different doses and different kinds of Low Molecular Weight Heparin (LMWH) on clot rate (CR), and to determine whether the CR can be used to monitor LMWH.

Methods This study was performed in two phases in vitro. The first, $\mathrm{CR}$ was measured with different reagent (glass beads, celite and kaolin) on volunteer $(\mathrm{n}=20)$ blood samples spiked with increasing concentrations of LMWH (dalteparin, $0.2-1.8 \mathrm{IU} / \mathrm{ml}$ ). The second, $\mathrm{CR}$ were measured with the same reagent (glass beads) on volunteer $(n=20)$ blood samples spiked with the same concentrations (0.8 IU/ $\mathrm{ml}$ ) of different LMWH (dalteparin, enoxaparin and nadroparin). Regression analysis was performed to establish a regression equation from corresponding anti-Xa LMWH levels.

Results With the increase in doses of dalteparin, CR values were reduced gradually and an exponential relationship was observed between the CR values and dalteparin concentrations $(p<0.01)$ for all three reagents. With the same concentrations $(0.8 \mathrm{IU} / \mathrm{ml})$ of LMWH, dalteparin showed a more effect on CR (dalteparin $7.4 \mathrm{IU} /$ min vs enoxaparin $8.5 \mathrm{IU} / \mathrm{min}$, nadroparin $8.5 \mathrm{IU} / \mathrm{min}, \mathrm{p}<0.05$ ). Compare with the baseline (17.6 IU/min), all three kinds of $\mathrm{LMWH}$ induced a significant change in the CR $(p<0.01)$.

Conclusions The in vitro studies have shown that, there was an exponential relationship between the CR and dalteparin concentrations for all three reagents. All three kinds of LMWH could significantly reduce the value of $\mathrm{CR}$. CR test may be a suitable method for monitoring the anticoagulant effect of LMWH.

\section{e0170 THE SENSITIVITY OF DIFFERENT REAGENTS FOR LABORATORY MONITORING OF LOW MOLECULAR WEIGHT HEPARIN: AN IN VITRO STUDY}

doi:10.1136/hrt.2010.208967.170

Shi Xu-Bo, Hu Da-Yi, Wang Jian-Qi, Chen Zhe, Yu Hai-Rong, Bai Ying. Department of Cardiology, Beijing Tong Ren Hospital, Beijing, China

Objective Because of the lack of Point-of-care testing, the use of low-molecular-weight heparin (LMWH)therapy in some special 
patients is restricted. This study was designed to determine the in vitro sensitivity to $\mathrm{LMWH}$ of different reagents by sonoclot analyser, and to determine whether the ACT can be used to monitor LMWH.

Methods This study was performed in vitro. ACT was measured with different reagents (glass beads, celite, and kaolin) on volunteer $(n=30)$ blood samples spiked with increasing concentrations of LMWH (dalteparin, 0.2-1.8 IU/ml). Linear regression analysis was performed to establish a regression equation from different concentration of dalteparin and corresponding ACT values.

Results Analysis of dose-response curves obtained in vitro, an excellent linear relationship was observed between the ACT and dalteparin concentrations for all three reagents $(p<0.01)$. Differences in slope of the regression curves of ACT were observed with all the reagents tested (glass beads $249.7 \mathrm{~s} / \mathrm{IU}$, celite $77.7 \mathrm{~s} / \mathrm{IU}$, and kaolin $59.3 \mathrm{~s} / \mathrm{IU}, \mathrm{p}<0.01)$. Reagents vary widely in their in-vitro sensitivity to dalteparin. In the concentration range of $0.2-1.8 \mathrm{IU} / \mathrm{ml}$, the gaolin reagent was too insensitive to dalteparin, and glass beads was the most suitable reagent for monitoring the anticoagulant effect of dalteparin.

Conclusions Using sonoclot analyser, there was an excellent linear relationship between the ACT and dalteparin concentrations for all the three reagents (glass beads, celite, and kaolin) in vitro. Glass beads may be a suitable reagent of ACT test for monitoring the anticoagulant effect of LMWH.

\section{e0171 THE SENSITIVITY OF NEW REAGENTS FOR LABORATORY MONITORING OF LOW MOLECULAR WEIGHT HEPARIN: AN IN VITRO STUDY}

doi:10.1136/hrt.2010.208967.171

Shi Xu-Bo, Hu Da-Yi, Wang Ji-Yun, Hou Xiao-Xia, Ma Zhi-Min. Department of Cardiology, Beijing Tong Ren Hospital, Beijing, China

Objective Low molecular weight heparin (LMWH) is currently the most commonly used intravenous anticoagulant drugs, but the lack of point of care testing (POCT) limit its applications in patients with severe renal dysfunction and others. The purpose of this study was to explore the sensitivity of new activated clotting time (ACT) reagents for laboratory monitoring of LMWH.

Methods Blood samples collected from 30 healthy volunteers. After taking blood samples, different doses of low molecular weight heparin (dalteparin) were added and the anti-Xa level of final blood samples was $0.1-1.8 \mathrm{IU} / \mathrm{ml}$. ACT and clot rate (CR) were measured with traditional reagent kaolin and new reagent magbar, Linear regression analysis was performed and a regression equation was established between different anti-factor Xa levels and the corresponding ACT, CR values.

Results With dalteparin concentration increased, the ACT values were gradually extended and the CR values were gradually reduced with both two reagents (kaolin and magbar). Analysis of doseresponse curves obtained in vitro, an excellent linear relationship was observed between the ACT and dalteparin concentrations for all two reagents $(p<0.01)$, and an exponential relationship was observed between the CR and dalteparin concentrations $(p<0.01)$. Differences in slope of the regression curves of ACT were observed with the reagents tested (magbar $1097.6 \mathrm{~s} / \mathrm{IU}$ vs kaolin $59.3 \mathrm{~s} / \mathrm{IU}$, $\mathrm{p}<0.01$ ).

Conclusions This in vitro study has shown that the sensitivity of traditional ACT test reagent (kaolin) for laboratory monitoring of dalteparin was poor, and the sensitivity of new ACT test reagents (magbar) for laboratory monitoring of dalteparin increased significantly. The new reagents magbar may be used for bedside monitoring of anticoagulant activity of LMWH.

\section{Basic Science: Experiment Research e0172 EFFECTS OF EPO ON PROLIFERATION OF RABBIT BONE MARROW MESENCHYMAL STEM CELLS}

doi:10.1136/hrt.2010.208967.172

${ }^{1}$ Li Zhengzhang, ${ }^{2}$ Cheng Yingzhang, ${ }^{1}$ Sehn Zhe, ${ }^{1}$ Xue Haoping, ${ }^{1}$ Chen Shi. ${ }^{1}$ Department of Cardiology, GaoYou people's Hospital, Jiangsu, China; ${ }^{2}$ Second Affiliated Hospital of Nanchang University School of Medicine, Nanchang, China

Objective To explore effects of erythropoietin (EPO) on proliferation, activity and cell cycle of rabbit bone marrow mesenchymal stem cells (BMSCs).

Methods A total of four healthy male New Zealand white rabbits aged 6 weeks were supplied by the Experimental Animal Center of Nachang University. Rabbit BMSCs were in vitro isolated by the density gradient centrifugation. Cells were assigned into normal, $2 \mathrm{u} /$ $\mathrm{ml}, 4 \mathrm{u} / \mathrm{ml}, 8 \mathrm{u} / \mathrm{ml}, 16 \mathrm{u} / \mathrm{ml}$ groups. In the normal group, cells received normal culture, without additional EPO stimulation. Following EPO intervention, cell morphology and growth were observed. Cell proliferation was detected by MTT assay. Cell cycle changes were measured by flow cytometry. Cell supernatant was collected to detect mass concentration of matrix metalloproteinase-2 (MMP-2).

Results EPO to intervene BMSCs 3d, cells had no abnormal changes, showing colony growth. With increase EPO concentration, cell absorbance gradually increased, especially in the $16 \mathrm{u} / \mathrm{ml}$ group. $(\mathrm{F}=28.029, \mathrm{p}=0.008)$. Compared with the normal group, cell cycle changed in the $2 \mathrm{u} / \mathrm{ml}, 4 \mathrm{u} / \mathrm{ml}, 8 \mathrm{u} / \mathrm{ml}, 16 \mathrm{u} / \mathrm{ml}$ groups, and proliferation index significantly increased $(p<0.05$ or 0.01$)$. Mass concentration of MMP-2 in supernatant was the lowest in the $16 \mathrm{u} /$ $\mathrm{ml}$ group, and the highest in the normal group. Significant differences were detected among groups $(t=213.21, p<0.001)$.

Conclusion EPO can increase the proliferative ability of BMSCs.

\section{e0173 ISOLATION CULTURE AND IDENTIFICATION OF RAT MSC}

doi:10.1136/hrt.2010.208967.173

${ }^{1}$ Li Xueyuan, ${ }^{2}$ Zhang Yang, ${ }^{1}$ Qi Guoxian. ${ }^{1}$ The First Hospital of China Medical University, Shenyang, Liaoning, China; ${ }^{2}$ The Centrol Affiliated Hospital of Shenyang Medical College, Shenyang, Liaoning, China

Aims To investigate different ways of isolating and culturing rat MSC and different serum concentrations of medium for the best selection. Materials and methods Direct adherence and density gradient centrifugation methods are used in MSC isolation, routine and modicum medium change methods are used in MSC culture. We compared the growth state, cell quantity and population doubling time of MSC under different culturing ways and different serum concentration medium such as $10 \%, 11 \%$ and $15 \%$. We identified cultured MSC in logarithmic growth phase (P3 generation) by cell surface antigen and its inducing differentiation function.

Results 4 methods, which are direct adherent and routinely changing of medium method, direct adherent and modicum medium changing method, density gradient centrifugation and routinely changing of medium method and density gradient centrifugation and modicum medium changing method, are used during MSC isolating and culturing respectively. The cellular average doubling time is $36.0 \pm 0.9 \mathrm{~h}, 23.5 \pm 1.1 \mathrm{~h}, 49.8 \pm 12 \mathrm{~h}$ and $48.0 \pm 0.8 \mathrm{~h}$ respectively There are cellular colonies forming 3 to 10 days after isolation, shaping like whirlpool. From the serum concentration screening experiment, we find that $11 \%$ is the most suitable one for MSC growth. The result of cell surface antigen identification of MSC through immunol histochemistry is CD45 (-), CD90 (+), and CD45 $0.38 \%$, CD90 $98.4 \%$ for positive expression of MSC through flow cytometry. MSC can be successfully induced to differentiate to chondrocyte and lipocyte. 